\title{
Stability of Collinear Points in the Generalized Photogravitational Robes Restricted Three-Body Problem
}

\author{
AbdulRazaq AbdulRaheem \\ Department of Mathematics Ahmadu Bello University Zaria, Nigeria \\ E-mail: raz11ng@yahoo.com \\ Received December 30, 2010; revised February 20, 2011; accepted February 23, 2011
}

\begin{abstract}
In studying the effects of radiation and oblateness of the primaries on the stability of collinear equilibrium points in the Robes restricted three-body problem we observed the variations of the density parameter $k$ with the mass parameter $\mu$ for constant radiation and oblateness factors on the location and stability of the collinear points $L_{1}, L_{2}$ and $L_{3}$. It is also discovered that the collinear points are unstable for $k>0$ and stable for $k<$ 0 .
\end{abstract}

Keywords: Stability, Collinear Points, Robes Problem, Density Parameters

\section{Introduction}

The classical Restricted Three-Body problem has been generalized in various forms $([1,11,13,12,5-7,10,14])$ by incorporating perturbing parameters such as perturbations in Coriolis and centrifugal forces, radiation pressure forces, oblateness of the primaries, drag effects and so on.

Robe [9] introduced a new kind of restricted three-body problem that incorporates the effect of buoyancy force. Here, one of the primaries is a rigid shell of mass filled with homogeneous incompressible fluid of density $\rho_{1}$. The second primary $m_{2}$ is a point mass located outside the shell. The third body $m_{3}$ is the particle of negligible mass of density $\rho_{3}$ which moves inside the shell under the influences of the gravitational attraction of the primaries and the buoyancy force of the fluid of density $\rho_{1}$. Robe studied the motion of the infinitesimal mass when $m_{2}$ describes both circular and elliptical orbits for $\rho_{1}=\rho_{3}$, obtained an equilibrium point at the centre of the shell and studied the linear stability of the point corresponding to the particular solution of the rotating system.

Recently, some forms of perturbations and other destabilizing parameters have been introduced to define new problems in Robes problem. Shrivastava and Garain [12] studied the effect of small perturbations in the Coriolis and centrifugal forces on the location of equilibrium points in the Robes problem. Plastino and Plastino [8] considered the Robes problem when the shape of the rigid shell is taken as Roche's ellipsoid to study the lin- ear stability of the equilibrium solutions. Giordano et al. [2] discussed the effect of drag force on the existence and stability of the equilibrium points in the Robes problem. Hallan and Rana [3] studied the existence of equilibrium points in the Robes problem and established that there exists one equilibrium in the Robes elliptical problem while the Robes circular problem has many equilibrium points. Hallan and Rana [4] studied the effect of oblateness on the location and stability of equilibrium points in the Robes circular problem when the primary other than the elliptical shell is oblate spheroid and proved that the center of the first primary is always an equilibrium point for all values of the density parameter $k$ and the mass parameter $\mu$.

Robes model is important for studying and understanding the problem of small oscillation that takes place within the core of a planet.

In our model, we consider a rigid shell which is oblate spheroid and the second primary radiating to study the stability of collinear equilibrium points in Robes restricted three-body problem while observing the variation of the location of the collinear points with the density parameter $k$ and the mass parameter $\mu$.

\section{Equations of Motion}

Let the mass of the rigid shell and the second primary be $m_{1}$ and $m_{2}$ respectively. Let the density of the incompressible fluid inside the shell be $\rho_{1}$ and that of the infinitesimal mass moving inside the shell be $\rho_{3}$ and its mass. Let $A$ denote the oblateness coefficient of the first 
primary such that $0<A \ll 1$ and $q$ the radiation force of the second primary, which is given by $F_{p}=F_{g}(1-q)$ such that $0<1-q \ll 1$.

Let $M_{1}, M_{2}$ and $M_{3}$ be the centers of $m_{1}, m_{2}$, and $m_{3}$ respectively such that $M_{1} M_{3}=r_{1}$ and $M_{2} M_{3}=r_{2}$. Let $G$ be the gravitational constant and $(x, y, z)$ the coordinates of the infinitesimal mass $m$. Further, we suppose that the line joining $m_{l}$ and $m_{2}$ is the x-axis. The total potential acting at $m$ is

$$
-\frac{G m_{2} q}{r_{2}}+\frac{4}{3} \pi G \rho_{1}\left(1-\frac{\rho_{1}}{\rho_{3}}\right) r_{1}^{2}--\frac{G m_{1}}{r_{1}}-\frac{G m_{1} A}{2 r_{1}^{3}},
$$

where

$$
\begin{aligned}
& r_{1}^{2}=\left(x-x_{1}\right)^{2}+y^{2}+z^{2} \\
& r_{2}^{2}=\left(x-x_{2}\right)^{2}+y^{2}+z^{2}
\end{aligned}
$$

The coordinates $m_{1}$ and $m_{2}$ are $\left(x_{1}, 0\right)$ and $\left(x_{2}, 0\right)$ respectively. In dimensionless rotational coordinate system, we choose the unit of mass to be the sum of the masses of the primaries, i.e. $m_{1}=1-\mu$ and $m_{2}=\mu$. The unit of length is taken as equal to the distance between the primaries, and the unit is chosen so that $G=1$.

The equations of motion of the infinitesimal body are

$$
\begin{gathered}
\ddot{x}-2 n \dot{y}=\Omega_{x} \\
\ddot{y}+2 n \dot{x}=\Omega_{y} \\
\ddot{z}=\Omega_{z} \\
\Omega=\frac{1}{2} n^{2}\left(x^{2}+y^{2}\right)-k r_{1}^{2}+\frac{1-\mu}{r_{1}}+\frac{\mu q}{r_{2}}+\frac{1-\mu}{2 r_{1}^{3}} A \\
k=\frac{4}{3} \pi G \rho_{1}\left(1-\frac{\rho_{1}}{\rho_{3}}\right), \mu=\frac{m_{2}}{m_{1}+m_{2}}, 0<\mu \leq \frac{1}{2} \\
n^{2}+1+\frac{3}{2} A \\
r_{1}^{2}=(x+\mu)^{2}+y^{2}+z^{2} \\
r_{2}^{2}=(x-1+\mu)^{2}+y^{2}+z^{2}
\end{gathered}
$$

\section{Equilibrium Points}

Equilibrium points exist for the system when

$$
\Omega_{x}=\Omega_{y}=\Omega_{z}=0 .
$$

For $k \neq 0\left(\right.$ i.e. $\left.\rho_{1} \neq \rho_{3}\right)$

$$
\begin{aligned}
& \Omega_{x}=n^{2} x-2 k(x+\mu)-\frac{(1-\mu)(x+\mu)}{r_{1}^{3}}-\frac{\mu q(x-1+\mu)}{r_{2}^{3}}-\frac{3}{2} \frac{(1-\mu)(x+\mu) A}{r_{1}^{5}} \\
& \Omega_{y}=n^{2} y-2 k y-\frac{(1-\mu) y}{r_{1}^{3}}-\frac{\mu q y}{r_{2}^{3}}-\frac{3}{2} \frac{(1-\mu) y A}{r_{1}^{5}} \\
& \Omega_{z}=2 k z-\frac{(1-\mu) z}{r_{1}^{3}}-\frac{\mu q z}{r_{2}^{3}}-\frac{3}{2} \frac{(1-\mu) z A}{r_{1}^{5}} .
\end{aligned}
$$

\section{Collinear Points}

Collinear equilibrium points occur when

$$
\Omega_{x}=\Omega_{y}=\Omega_{z}=0 \text { and } y=0, z=0 .
$$

The equation

$$
\begin{aligned}
f(x)= & n^{2} x-2 k(x+\mu)-\frac{(1-\mu)(x+\mu)}{r_{1}^{3}} \\
& -\frac{\mu q(x-1+\mu)}{r_{2}^{3}}-\frac{3}{2} \frac{(1-\mu)(x+\mu) A}{r_{1}^{5}}
\end{aligned}
$$

gives the positions of the collinear points. We observe that $f(-\infty)<0, f(\mu-1)>0, f(\mu)<0$ and $f(\infty)>0$ so that the collinear points $L_{1}, L_{2}$ and $L_{3}$ lie in the intervals $(\mu, \infty),(\mu-1, \mu)$ and $(-\infty, \mu-1)$ respectively.

For $L_{1}$, put $r_{1}=\mu-x_{1}, r_{2}=x_{1}-1+\mu$ and substituting these values of $r_{1}$ and $r_{2}$ in Equation (8), putting $\xi_{1}=x_{1}-1+\mu$ we obtain

$$
\begin{aligned}
& {[2+3 A-4 K] \xi_{1}^{7}+[(2+3 A)(5-\mu)-20 k] \xi_{1}^{6}} \\
& +[(2+3 A)(10-4 \mu)-40 k] \xi_{1}^{5} \\
& +[(2+3 A)(10-6 \mu)+2 \mu-2 \mu q-2-40 k] \xi_{1}^{4} \\
& +[(2+3 A)(5-4 \mu)+4 \mu-8 \mu q-4-20 k] \xi_{1}^{3} \\
& -4[3 \mu q+k] \xi_{1}^{2}-8 \mu q \xi_{1}-2 \mu q=0
\end{aligned}
$$

This is a seventh degree algebraic equation in $\xi_{1}$. For both positive and negative values of $\mathrm{k}$ there is only one change of sign in the equation. This shows the existence of at least one real root. Similarly, we have for $L_{2}$ and $L_{3}$

$$
\begin{aligned}
& {[2+3 A-4 K] \xi_{2}^{7}+[(2+3 A)(5-\mu)-20 k] \xi_{2}^{6}} \\
& +[(2+3 A)(10-4 \mu)-40 k] \xi_{2}^{6} \\
& -[(2+3 A)(10-6 \mu)+2 \mu-2 \mu q-2-40 k] \xi_{2}^{4} \\
& +[(2+3 A)(5-4 \mu)+4 \mu-8 \mu q-4-20 k] \xi_{2}^{3} \\
& -4[3 \mu q+k] \xi_{2}^{2}-8 \mu q \xi_{2}-2 \mu q=0 .
\end{aligned}
$$

and 


$$
\begin{aligned}
& {[2+3 A-4 K] \xi_{3}^{7}+[(2+3 A)(2+\mu)-8 k] \xi_{3}^{6}} \\
& +[(2+3 A)(1+2 \mu)-4 k] \xi_{3}^{5}-(2+3 A+2 \mu q) \xi_{3}^{4} \\
& +2(2-2 \mu) \xi_{3}^{3}-(2+3 A)(1-\mu) \xi_{3}^{2} \\
& -6 A(1-\mu) \xi_{3}-3 A(1-\mu)=0 .
\end{aligned}
$$

where $\xi(i=1,2,3)$ are given by Equations (9), (10) and (11) respectively for $\xi_{2}=1-\mu-x_{2}$ and $\xi_{3}=\mu+x_{3}$. Therefore, the three collinear points are located as

$$
\begin{aligned}
& x_{1}=1-\mu+\xi_{1} \\
& x_{2}=1-\mu+\xi_{2} \\
& x_{3}=\xi_{3}-\mu
\end{aligned}
$$

Using MATLAB we generated real roots $\xi_{i}(i=1,2,3)$ of the Equations (9), (10) and (11) respectively to compute the respective values $x_{1}(i=1,2,3)$ of the collinear points.

For constant values of the oblateness factor $\mathrm{A}$ and the radiation factor q, there is only one real root of (9) for each $=0,1,3,5$ and $\mu=0.1,0.2,0.3,0.4,0.5$, three real roots of (10) and (11) except for $k=1$ where there are five distinct real roots in the case of Equation (11).

The variation in values of for various values of the density parameter $k$ and mass parameter $\mu$ is shown in Figures 1, 2 and 3 respectively.

In Figures 1-3, the curve $k=0$ corresponds to $\rho_{1}=\rho_{3}$. That is, when the density of the fluid in the rigid shell is same as the density of the infinitesimal mass moving in the shell. The other curves, and correspond to the case when $k=1, k=3, k=5$ correspond to the case when $\rho_{3}>\rho_{1}$.

\section{Stability of Collinear Points}

Let the equilibrium points and their positions be denoted by $L\left(x_{0}, y_{0}\right)$.Then we substitute

$$
x=x_{0}+\xi \text { and } y=y_{0}+\eta
$$

into the equations of motion (1) and obtain the equations of variation as

$$
\begin{aligned}
& \ddot{\xi}-2 n \dot{\eta}=\Omega_{x x}\left(x_{0}, y_{0}\right)+\Omega_{x y}\left(x_{0}, y_{0}\right) \\
& \ddot{\eta}+2 n \dot{\xi}=\Omega_{x y}\left(x_{0}, y_{0}\right)+\Omega_{y y}\left(x_{0}, y_{0}\right)
\end{aligned}
$$

where $\xi$ and $\eta$ are small displacement in $x_{0}$ and $y_{0}$ respectively. Retaining only linear terms in $\xi$ and $\eta$ the characteristic Equations of (13) is

$$
\lambda^{4}+\left(4 n^{2}-\Omega_{x x}^{0}-\Omega_{y y}^{0}\right) \lambda^{2}+\Omega_{x x}^{0} \Omega_{y y}^{0}-\left(\Omega_{x y}^{0}\right)^{2}=0
$$

where the superscripts indicate that the partial derivatives of $\Omega$ are to be evaluated at the equilibrium points $\left(x_{0}, y_{0}\right)$

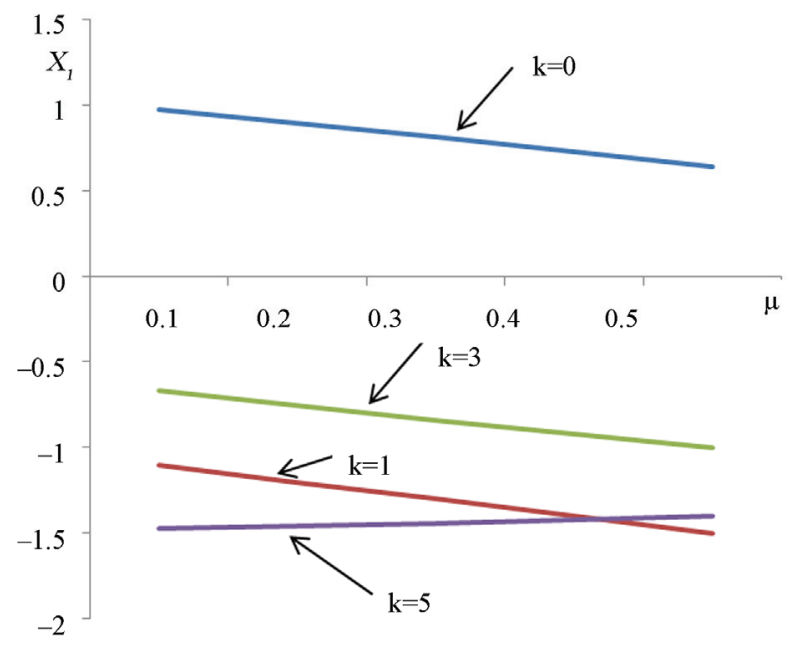

Figure 1. Variation of location of $L_{1}$ with density parameter $\boldsymbol{k}$ and mass parameter $\mu$.

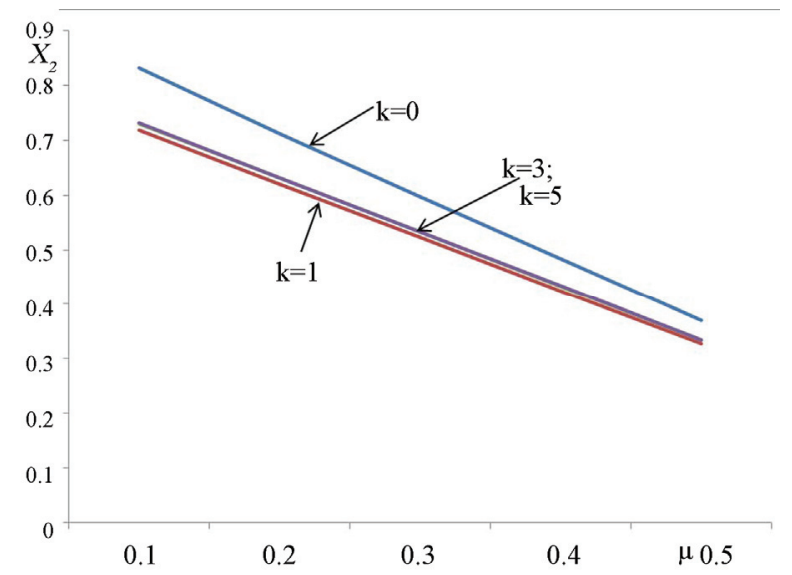

Figure 2.Variation of location of L2 with density parameter $k$ and mass parameter $\mu$.

At the collinear point $y=0, z=0$, so that $r_{1}^{2}=$ $(x+\mu)^{2}$ and $r_{2}^{2}=(x-1+\mu)^{2}$. Therefore,

$$
\begin{aligned}
& \Omega_{x x}^{0}=n^{2}-2 k+\frac{2(1-\mu)}{r_{1}^{3}}-\frac{2 \mu q}{r_{2}^{3}}-\frac{6(1-\mu) A}{r_{1}^{5}}>0 \\
& \Omega_{x y}^{0}=0 \\
& \Omega_{y y}^{0}=n^{2}-2 k+\frac{1-\mu}{r_{1}^{3}}-\frac{\mu q}{r_{2}^{3}}-\frac{3}{2} \frac{(1-\mu) A}{r_{1}^{5}}<0 .
\end{aligned}
$$

For values of $\mu<\frac{1}{2}, 1-q \square 0, A \square 1$ the discriminant of the characteristic Equation (14) is positive when $k>0$ and negative for $k<0$.

Consequently, for $k>0$, one root $\lambda^{2}$ of the characteristic equation will be positive and the other negative, indicating unbounded motion. This makes the collinear 


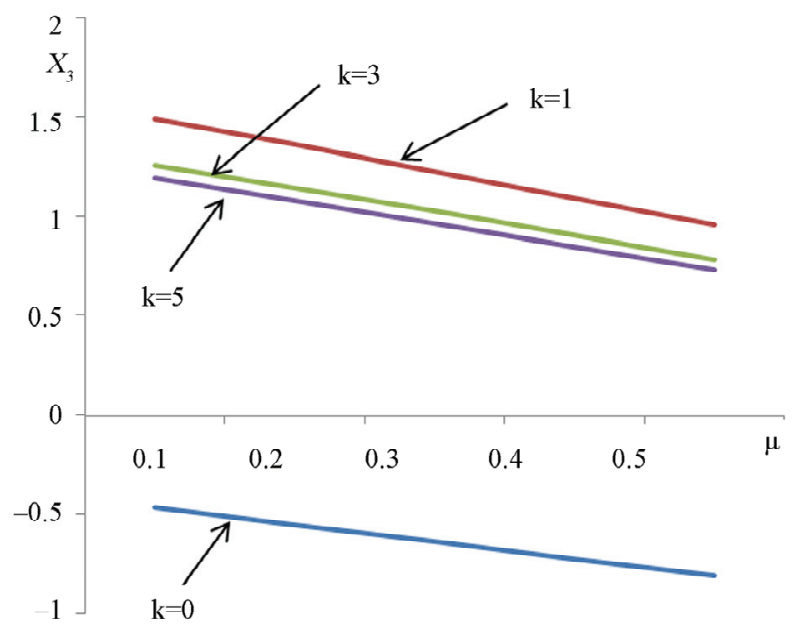

Figure 3. Variation of location of $\mathrm{L3}$ with density parameter and mass parameter $\mu$.

points $L_{i}(i=1,2,3)$ unstable. For $k<0$, both roots of the characteristic equation will complex (imaginary) and indicates oscillatory motion. Hence, the collinear points $L_{i}(i=1,2,3)$ are stable.

\section{Discussion}

It is evident from Equations (9), (10) and (11) that the three physical parameters (density, radiation and oblateness) affect the location of collinear points. Using numerical values we obtained nine collinear equilibrium points for our problem (one from Equation (9), three from Equation (10) and five from Equation (11). By keeping the radiation and oblateness factors constant, we observed the variation in the location of the collinear points for various values the density parameter with the mass parameter.

In Figures 1-3, curve $k=0$ corresponds to the density of the incompressible fluid in the spherical shell and that of the infinitesimal mass moving in the shell being the same. Curves $k=1, k=3$ and $k=5$ correspond to the infinitesimal mass denser than the incompressible fluid in the shell. It is also observed that due to variation of the parameter, keeping radiation and oblateness fixed, the positions of the collinear equilibrium points shifted towards the primaries. Hence, it could be inferred that the three physical parameters have the tendency of moving the collinear points closer to the primaries.

Collinear equilibrium points are generally unstable in classical and some generalized restricted three-body problem. However, it is seen from the discriminant of the characteristic Equation (14) that when the infinitesimal mass is denser than the incompressible fluid in the shell the collinear equilibrium points are unstable and stable when the incompressible fluid is denser than the infinitesimal mass.

\section{Conclusions}

In studying the stability of collinear equilibrium points in Robe's restricted three-body problem under the influence of radiation and oblateness of the primaries when the densities of the infinitesimal mass and the incompressible fluid are different nine collinear points were obtained.

The collinear points are found to be stable when the incompressible fluid in the spherical rigid shell is denser than the infinitesimal mass moving in the shell and unstable when the infinitesimal mass is denser than the incompressible fluid.

\section{References}

[1] A. AbdulRaheem and Singh, "Combined Effects of Perturbations, Radiation and Oblateness on the Stability of Equilibrium points in the Restricted Three-Body Problem," Astronomical Journal, Vol. 131, No. 3, 2006, pp. 1880-1885.

[2] R. K. Sharma, Z. A. Taqvi and K. B. Bhatnagar, Celest. Mech. and Dyn. Astr., Vol. 79, 2001, pp. 119-133.

[3] J. Singh, Indian J. of Pure and Appl. Math., Vol. 34, No. 2, 2003, pp. 335-345.

[4] S. K. Shaoo and B. Ishwar, Bull. of Astr. Society. of India, Vol. 28, 2000, pp. 579-586.

[5] B. Ishwar and A. Elipe, Astrophysics and Space Science, Vol. 277, 2001, pp. 437-446.

[6] A. L. Kunisyn, Jour. of Appl. Math. and Mech., Vol. 64, No. 5, 2000, pp. 757-763.

[7] A. L. Kunisyn, Jour. of Appl. Math. and Mech., Vol. 65, No. 4, 2001, pp. 703-706.

[8] D. V. Schuerman, Astronomical Journal, Vol. 238, 1980, pp. 337-342.

[9] V. V. Szebehely, Astronomical Journal, Vol. 72, No. 1, 1967, pp. 7-9.

[10] H. A. G. Robe, Celestial Mechanics, Vol. 16, 1977, pp. 345-351.

[11] A. K. Shrivastava and D. Garain, Celest.and Mech. Dyn. Astr., Vol. 51, 1991, pp. 67-73.

[12] C. M. Giordano, A. R. Plastino and A. Plastino, Celest. Mech. and Dyn. Astr., Vol. 66, 1997, pp. 229-242.

[13] P. P. Hallan and N. Rana, Celest . Mech.and Dyn. Astr., Vol. 66, 2001, pp. 145-155.

[14] P. P. Hallan and N. Rana, Indian J. of Pure and Appl. Math., Vol. 35, No. 3, 2004, pp. 401-413. 